\title{
Towards molecular computing: Co-development of microfluidic devices and chemical reaction media
}

\author{
Philip H. King, Josephine C. Corsi, Ben-Hong Pan, Hywel Morgan, Maurits R.R. de Planque, \\ Klaus-Peter Zauner*
}

Electronics and Computer Science E Institute for Life Sciences, University of Southampton, Southampton, SO17 1BJ, United Kingdom

\section{A R T I C L E I N F O}

\section{Article history:}

Received 15 December 2011

Accepted 4 January 2012

\section{Keywords:}

Microfluidics

Belousov-Zhabotinsky reaction

Chemical computing

Computing substrates

\begin{abstract}
A B S T R A C T
Microfluidics provides a powerful technology for both the production of molecular computing components and for the implementation of molecular computing architectures. The potential commercial applications of microfluidics drive rapid progress in this field-but at the same time focus interest on materials that are compatible with physiological aqueous conditions. For engineering applications that consider a broader range of physico-chemical conditions the narrow set of established materials for microfluidics can be a challenge. As a consequence of the large surface to volume ratio inherent in microfluidic technology the material of the device can greatly affect the chemistry in the channels of the device. In practice it is necessary to co-develop the chemical medium to be used in the device together with the microfluidic devices. We describe this process for a molecular computing architecture that makes use of excitable lipid-coated droplets of Belousov-Zhabotinsky reaction medium as its active processing components. We identify fluoropolymers with low melting temperature as a suitable substrate for microfluidics to be used in conjunction with Belousov-Zhabotinsky droplets in decane.
\end{abstract}

(c) 2012 Elsevier Ireland Ltd. All rights reserved.

\section{Molecular Computing}

Computing capability has advanced at a rapid pace since the introduction of the first commercially available integrated microprocessor circuit four decades ago. At the same time the underlying technology has changed surprisingly little. Increasingly, however, the limitations of information technology based on vast numbers of simple and fast solid-state switches becomes apparent. In applications where space or energy supply is severely restricted such as micro-robotics, smart drugs, smart materials, synthetic biology, bioimmersive computing, and controlled soft matter, solid-state computing is often not applicable. The disadvantages of conventional computing implementations are particularly apparent in comparison to natural information processing. Conversely, natural information processing systems point towards alternative modes of computing based on soft-matter rather than solid-state materials as computing substrate (Conrad, 1972; Zauner, 2005).

Nature's molecular computing architectures are highly organised heterogeneous chemical systems. The large surface-to-volume ratio concomitant with a high degree of compartmentalisation allows for fine-grained control of chemical reactions. Present engineering is far from achieving anything like the level of organisation

\footnotetext{
* Corresponding author.

E-mail address: kpz@ecs.soton.ac.uk (K.-P. Zauner).
}

found in nature. Nevertheless, the advent of microfluidics has in recent years provided a tool that facilitates better spatio-temporal control over chemical reaction media and thus enables new directions in the quest to engineer molecular computing devices (Jones et al., 2011). In practice, however, the large surface-to-volume ratio in microfluidic devices that confers the enhanced control over reactions, also has a downside: the material of the microfluidic devices can greatly affect the reactions that take place in the device channels and wells. The interactions between the reaction medium and the enclosing microfluidic device elements can be chemical or physical in nature. In the former case the chemical medium can alter the properties of the microfluidic device, in the latter the containing walls may act as a semipermeable surface and affect the concentration ratios of chemical species available in the reaction mixture. From this perspective, ideally the material of the microfluidic device would be as inert as possible. However, fabricating devices typically necessitates the reliable and strong bonding of several layers and the difficulty of achieving this increases with chemical inertness of the material.

As a consequence of the interaction between the chemical reaction medium and the microfluidic device itself, it is advantageous that both be developed in concert. The remainder of this paper will address the practice of device-reaction co-development. The specific setting to be discussed is a molecular computing architecture using lipid-coated droplets of the Belousov-Zhabotinsky (BZ) reaction medium in an oil phase. These droplets are excitable 
building blocks which are chemically coupled to form information processing networks. The droplets are prepared and arranged into communicating structures by means of a microfluidic chip. The chip is exposed to low $\mathrm{pH}$ and radicals from the harsh polar $\mathrm{BZ}$ medium. It is also exposed to the non-polar oil phase that fills the space on the chip not occupied by BZ-droplets. Consequently, the surface properties of the microfluidic chip material, the composition of the surfactant-containing oil phase and the aqueous droplets all have to be developed in concert. It is this aspect of addressing the challenge of molecular computing architectures that we focus on here.

\section{Materials for Microfluidics and Microdroplets}

Until recently, almost all microfluidic chips have been developed for aqueous biological solutions of neutral $\mathrm{pH}$, for example cell suspensions or blood serum samples for a diagnostic point-ofcare device. For biological and biomedical applications it is usually essential that all positions within the device can be interrogated with optical microscopy. As a result, materials for microfluidic devices have been selected primarily for three main properties: compatibility with mild aqueous solutions, optical transparency and ease of fabrication. The latter factor covers a wide variety of technical aspects; we just note here that it is favourable if the device structure can be formed from a polymerisable liquid precursor solution, and that the material should be able to bond to a flat surface. This is because most lithography-based fabrication methods only allow planar features (Gardner et al., 2001). Open trenches or open wells are thus created in the main (polymerised) material, which is then bonded to a flat surface to form enclosed channels and wells. Bonding with glue is an example where the device material should not degrade because of exposure to a non-aqueous solution and where the device components (i.e. uncured glue) should not leak into the microfluidic channels.

The above factors have led to the situation where the vast majority of polymer-based microfluidic devices reported in the literature (see Table 1) are based on polydimethylsiloxane (PDMS) (Duffy et al., 1998). PDMS is flexible, strong, optically transparent, gas permeable (aiding degassing), biocompatible and also easily and monolithically bonded using an oxygen plasma treatment process that avoids the use of glue (Haubert et al., 2006). The introduction of PDMS has greatly accelerated the pace of microfluidic research, as it can be processed by 'soft lithography' (i.e. from elastomeric polymers) without the use of expensive cleanroom facilities (Xia and Whitesides, 1998), as required for patterning glass and silicon ('hard lithography').

One approach for creating heterogeneous computing substrates is to compartmentalise the reaction mixture. In general, water-in-oil droplets in microfluidic devices have recently gained considerable interest for biological and chemical analysis (Christopher and Anna, 2007; Huebner et al., 2008; Theberge et al.,
2010). Handling analytes in compartmentalised droplets significantly reduces the required amount of reagents, prevents substance diffusion, reduces sample-device interactions and allows multiple samples to be analysed in parallel on the same chip. The aqueous droplets can be stabilised by adding a surfactant that self-assembles at the water-oil interface and prevents droplet coalescence.

Our goal is to engineer chemical computing substrates, using aqueous droplets of the Belousov-Zhabotinsky (BZ) medium as an oscillating chemical reaction. Networks of lipid-stabilised BZ droplets in oil can be brought into contact, with the transmission of oscillations mediated by the droplet-to-droplet interface (Corsi et al., 2011; Gorecki et al., 2011). This system is very different from a standard biological sample, and has required the co-development of the chemicals and the microfluidic device itself. PDMS swells considerably in non-polar solvents (Lee et al., 2003) such as oil, which causes microfluidic channels to deform to the extent that they can even become blocked. PDMS also absorbs small ions such as bromide (Ginn et al., 2004). This is a particularly undesired effect because the exact concentrations of these ions determines the oscillating properties of the BZ medium. Hence, PDMS cannot be used as a microfluidic chip material for the positioning of compartmentalised $\mathrm{BZ}$ and the characterisation of excitation through droplet networks.

\section{Co-development of Device and Chemical Medium}

The co-evolution of the device material with the chemical substrate has led to the development of a microfluidic-compatible variation on the BZ reaction. Traditionally, BZ uses malonic acid as the substrate. However, the breakdown of bromomalonic acid releases carbon dioxide. The release of gas within a microfluidic device, even one made of a gas-permeable material such as PDMS, is highly undesirable. Gas bubbles can block channels and are compressible, thus complicating the external control over the fluid flows. Furthermore, these negative effects are compounded when using alternative materials because these are typically not permeable to gas at all.

An alternative BZ-reaction substrate is 1,4-cyclo-hexanedione (CHD) (Corsi et al., 2011; Kurin-Csörgei et al., 1996). It displays different behaviour when compared to malonic acid based BZ, including a 4-h induction phase (as compared to 5-15 min for malonic acid), but this disadvantage is outweighed by the lack of noticeable gas production. This significantly simplifies the required microfluidic design by avoiding the need for bubble traps and similar structures. However, droplets of $\mathrm{BZ}$ in oil proved prone to coalesce, probably because the low $\mathrm{pH}$ of the $\mathrm{BZ}$ mixtures degrades the droplet-coating lipid molecules. Using lower acid concentrations in the $\mathrm{BZ}$ reaction solved this chemical incompatibility.

There have recently been a number of publications detailing alternatives to PDMS for microfluidic chip fabrication (Morel et al.,

Table 1

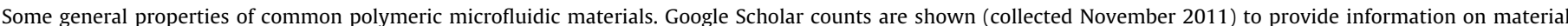
prevalence in the literature, and are normalised to the count for PDMS.

\begin{tabular}{|c|c|c|c|c|}
\hline & Biocompatible & Non-polar solvent resistant & Acid resistant & Google Scholar count \\
\hline PDMS & $\gamma$ & $X$ & $\checkmark$ & 100.0 \\
\hline PMMA & & $X$ & $\lambda$ & 38.5 \\
\hline Polyimide & & $\sqrt{ }$ & $\sqrt{ }$ & 17.9 \\
\hline Polystyrene & & 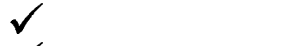 & $\sqrt{ }$ & 11.5 \\
\hline PVDF & & $\checkmark$ & $\sqrt{ }$ & 6.1 \\
\hline $\mathrm{COC}$ & & $X$ & & 5.7 \\
\hline PVC & $X$ & 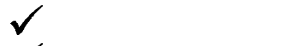 & $\sqrt{ }$ & 5.4 \\
\hline NOA81 & 1 & $\sqrt{ }$ & $?$ & 0.5 \\
\hline THV & & 1 & & 0.1 \\
\hline
\end{tabular}




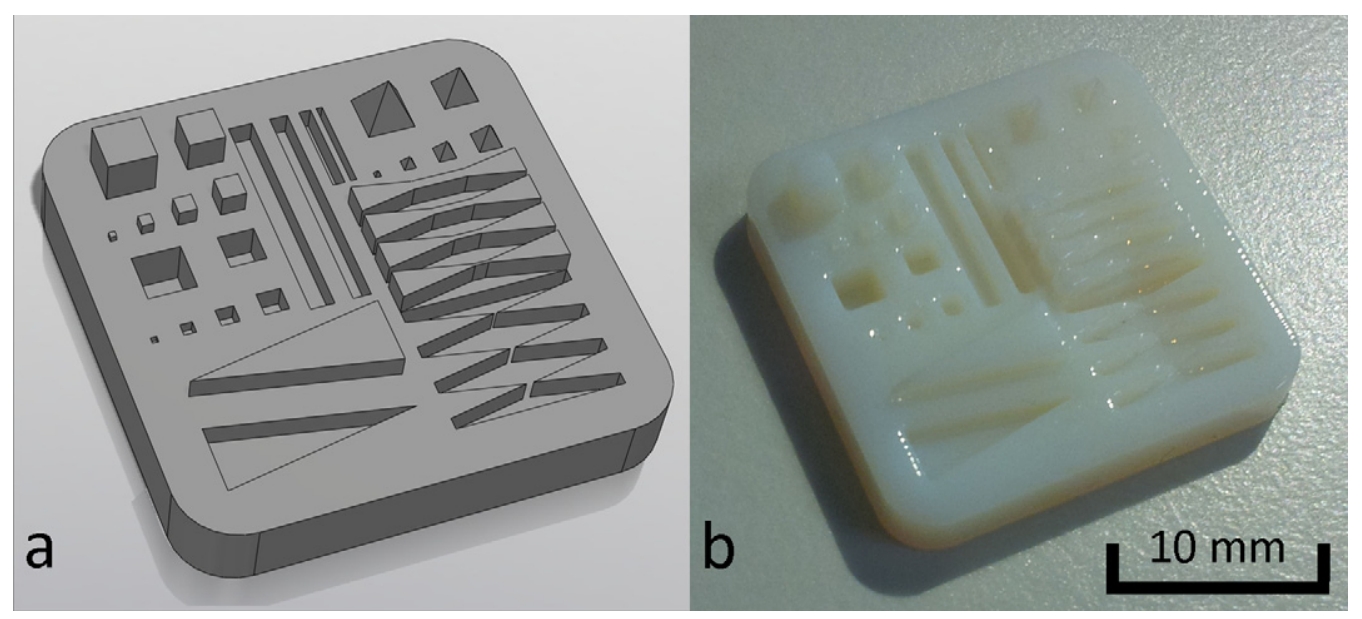

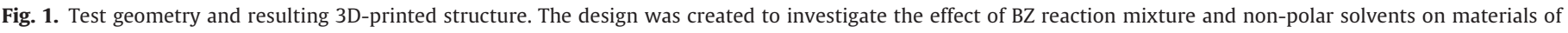
interest. Model created using SolidWorks 2009 (Dassault Systems, France) (a). 3D-printed structure created directly from the CAD geometry (b).

2009; Wägli et al., 2010). Norland Optical Adhesive 81 (NOA81) is a UV-curable glue, conventionally used for securing optical components such as lenses. It is gas impermeable, transparent, does not swell in organic solvents and, like PDMS, is bondable using oxygen plasma. However, it is less flexible and more hydrophilic than PDMS.

Fluoropolymers have for some time been of interest as substrates for microfluidics. Polytetrafluoroethylene (PTFE) is used extensively for chemically resistant coatings and tubing. However, their widespread use in microfluidics has been hindered by their high melting temperatures (for PTFE, $>300^{\circ} \mathrm{C}$ ) for moulding and embossing. A potential breakthrough could be found in a new fluoropolymer, Dyneon THV 500 (Begolo et al., 2011). A terpolymer of tetrafluoroethene, hexafluoropropylene and vinylidene fluoride, THV 500 has a melting point of $200^{\circ} \mathrm{C}$. This lower melting point allows the material to be processed in laboratory vacuum ovens, which usually have a peak temperature of around $200^{\circ} \mathrm{C}$.

Additive layer manufacture technologies, such as stereolithography, have been the subject of much research for the production of microfluidic systems (King and Covington, 2009; Han et al., 2005). One major technique is 3D printing (3DP), where multiple layers of a light-curable material are deposited by a modified inkjet printer head. Each layer is subsequently cured using a high-intensity lamp. 3D printing currently lacks the resolution of lithographic techniques, but is automated, fast and does not require cleanroom facilities. This has allowed the use of 3D-printing to create moulds for PDMS, without the need for lithographic processes at all (Bonyár et al., 2010; Huang et al., 2009). It may also be possible to create the microfluidic chips themselves with 3D printing, avoiding polymer casting and bonding processes altogether, but this would depend on the chemical compatibility of the 3D-printing material.

\section{Materials Testing for Compartmentalised BZ Medium}

To assess the chemical resistance of PDMS, NOA81, THV 500 and 3D-printing material, a test model was designed using a 3D CAD package, shown in Fig. 1. The features had a minimum linewidth of $200 \mu \mathrm{m}$, and heights of up to $3 \mathrm{~mm}$, and were designed so that chemical degradation could be easily identified as, for example, corner-rounding effects. The geometries were designed to be fabricated using a 3D printing system (Objet Connex350 ${ }^{\mathrm{TM}}$ ), and allow investigation of swelling, shrinkage or corrosive effects experienced by the materials. The 3D-printed structure was used directly for chemical compatibility studies of 3D-printer materials. For the other materials, in a normal soft lithography process, a negative of the depicted geometries was produced with the 3Dprinter. In this negative mould, PDMS was polymerised to obtain the PDMS test structure. For the NOA81 and THV test structures, a positive-negative-positive mould approach was then used: the first, positive 3D-printed structure was used to obtain a negative PDMS structure, which then served as a mould to obtain the desired THV and NOA81 patterns. PDMS is an elastomeric substrate, and this prevents deformations resulting from separating two inelastic materials (i.e. 3D-printed material and THV or NOA81).

The geometric structures fabricated from PDMS, the 3DP material (Objet Vero WhitePlus ${ }^{\mathrm{TM}}$, a proprietary acrylic material), NOA81 and THV 500 were exposed overnight to decane, a non-polar solvent (sometimes used in microfluidic systems) in which lipid molecules dissolve well. The higher molecular weight alkanes such as hexadecane are more commonly used within PDMS microfluidic devices but the lipid solubility is poor, preventing lipid-stabilisation of aqueous droplets.

The effect of the decane oil on PDMS is shown in Fig. 2. The PDMS has swollen by $20 \%$, whereas the remaining materials retained their original geometries. This clearly demonstrates that PDMS is unsuitable for BZ-in-oil microfluidic devices, whereas, at least in terms of deformation, the other materials are decane-compatible.

Another set of geometric structures, after exposure to $\mathrm{BZ}$ medium for 1,5 and $17 \mathrm{~h}$, and subsequent extensive washing with deionized water, are shown in Fig. 3. It can be concluded that the geometric structures of all four materials are not degraded by exposure to the BZ reactants. Micrographs of features on the THV structures, before and after exposure to $\mathrm{BZ}$ and oil, can be seen in Fig. 4. The NOA81 structure did shrink by around $1.3 \%$ for the longest incubation, but since the entire pattern was scaled down it is unlikely that this was due to chemical degradation. NOA81 is a photocurable material, and may thus shrink over time as further photocuring occurs. However, the materials used by the 3D printing system are also photocurable. The fact that no such effect was observed in the 3DP material, highlights the high quality of commercial (proprietary) material formulations.

What is noticeable across the range of materials is a change in colour. In the case of the NOA81, an initial bleaching from the original structure's yellow colour is followed by a continuous staining. The other three materials also experience staining over time, although the effect is less apparent in the fluoropolymer THV 500. It is possible that these colour changes are due to chemicals being absorbed into the material, or that BZ components precipitate over time on top of the test structures. 


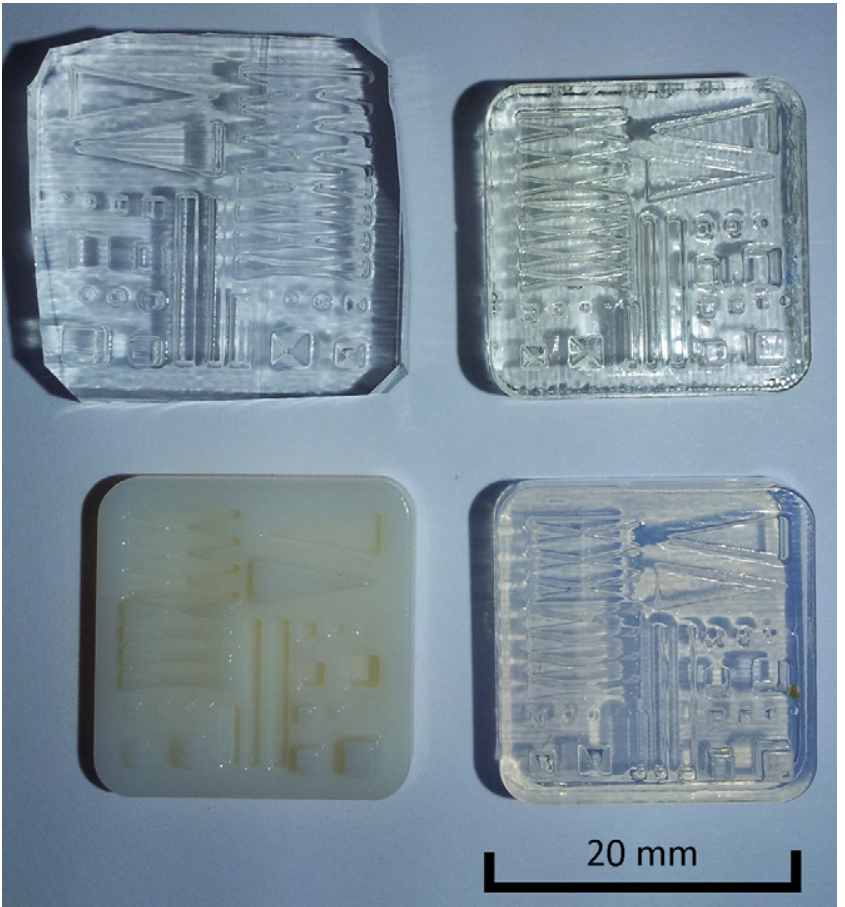

Fig. 2. Structures exposed to decane. The structures were exposed to decane, a nonpolar solvent for $17 \mathrm{~h}$. The PDMS (upper left) has absorbed the solvent, and has swollen by 20\%. The other structures (clockwise from PDMS: NOA81, THV 500, 3DP) appear unchanged.

When the test structures of the four materials were submerged in BZ medium in individual Petri dishes, the BZ oscillations were similar in all four dishes. However, when the BZ reagents were loaded into an open-well geometry (i.e. BZ medium enclosed in

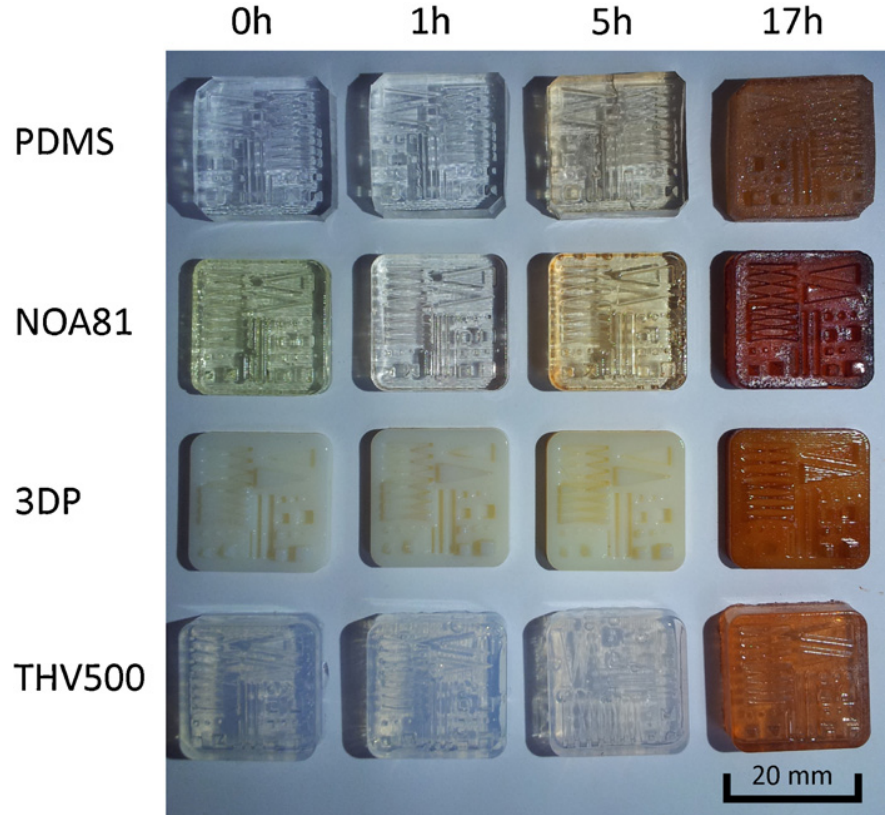

Fig. 3. Test structures exposed to $\mathrm{CHD} B Z$ reaction mixture for varying lengths of time, compared to original, untreated structures.

a channel and deeper well), as shown in Fig. 5, oscillations readily occurred in the THV channel and well, but not in the 3D-printed material. The 3DP material thus appears to inhibit the BZ oscillation at larger device-area-to-BZ-volume ratios, which could be due to absorption of $\mathrm{BZ}$ components into the 3D-printed material, or to molecules from the channels walls leaking into the $\mathrm{BZ}$ aqueous phase.
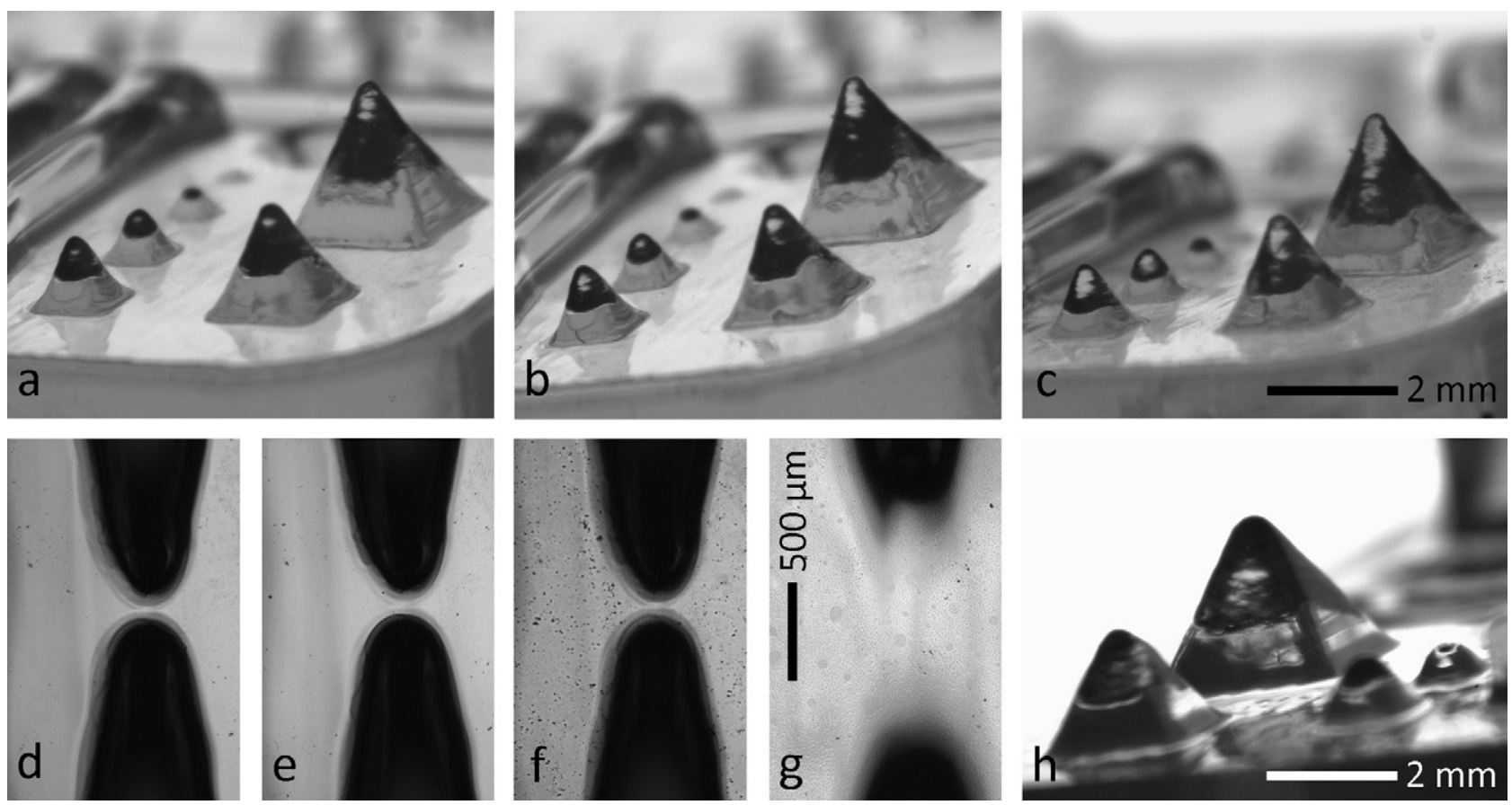

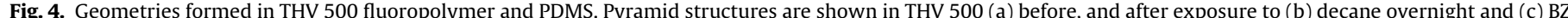

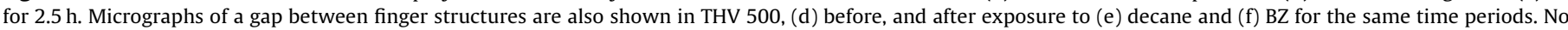

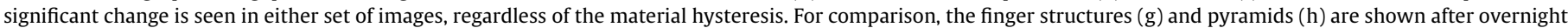

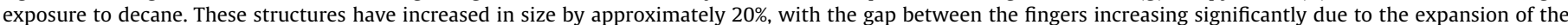

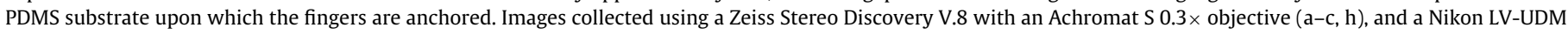
microscope with an LU Plan Fluor $5 \times$ objective $(\mathrm{d}-\mathrm{g})$. 


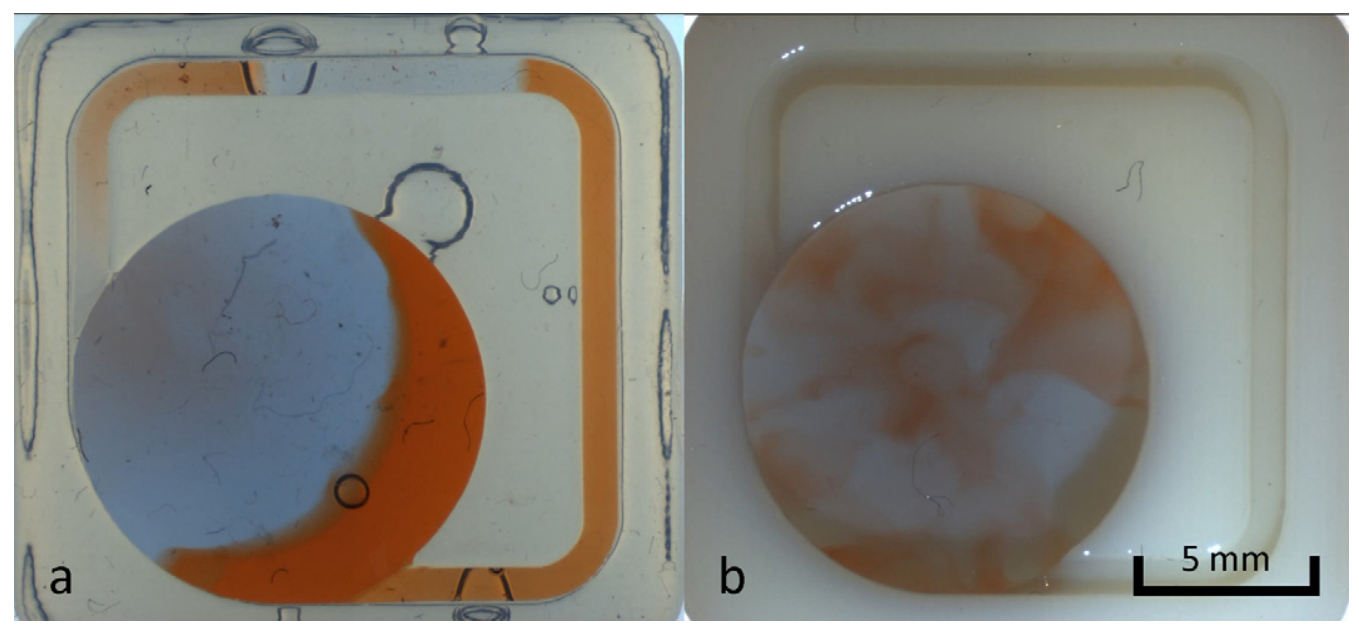

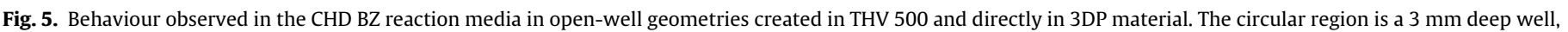

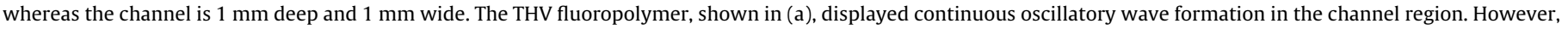
the 3DP material, shown in (b) does not show oscillatory behaviour, suggesting that it inhibits oscillation.

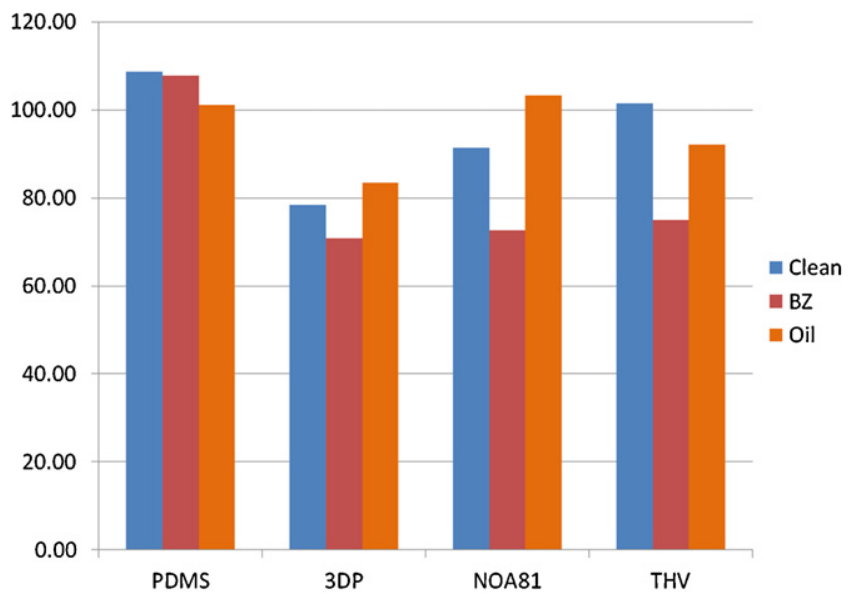

Fig. 6. Contact angle measurements obtained from flat test structures produced from PDMS, NOA81, 3DP material and THV 500. Measurements were obtained by placing a droplet of deionised water on non-exposed material, material exposed to CHD BZ for $5 \mathrm{~h}$, and material exposed to decane for $17 \mathrm{~h}$. The values obtained for the fresh material are close to those found in the literature for PDMS (Duffy et al., 1998), NOA81 (Wägli et al., 2010) and THV (Begolo et al., 2011)-no such data exists for the 3DP material.
Finally, the effect of the CHD BZ reaction mixture on the surface energy of the various materials was probed using contact angle measurements. The high surface area to volume ratio of microfluidic devices means that surface effects can be critical, and this is especially true in droplet-based systems. Ideally, all of the device surfaces should be hydrophobic, to minimise interactions between the device material and the aqueous phase. A change in the material contact angle could suggest damage to the material, which may be acceptable as long as the resultant surface energy is constant. For testing, flat surfaces of PDMS were created by replica moulding on top of glass slides. Again, THV and NOA81 were moulded upon a PDMS mould, whereas the 3DP material was printed directly.

The results of the contact angle measurements are shown in Fig. 6. PDMS appears to show the least variation in contact angle during the tests, along with the 3DP material. THV and NOA81 both show significant drops in their contact angle after exposure to the BZ substrate. Interestingly, for THV, washing the material after exposure to the $\mathrm{BZ}$ substrate appears to reverse the contact angle change caused by exposure to the BZ medium (Fig. 7). This could indicate that a layer of chemicals precipitates from the BZ mixture but is unable to penetrate the THV polymer. Experiments like this are important to establish whether BZ oscillations are suppressed because molecules from the BZ mixture are absorbed by the microfluidic device (depletion mechanism) or because interfering molecules are released from the device material into the BZ solution (inhibition mechanism).

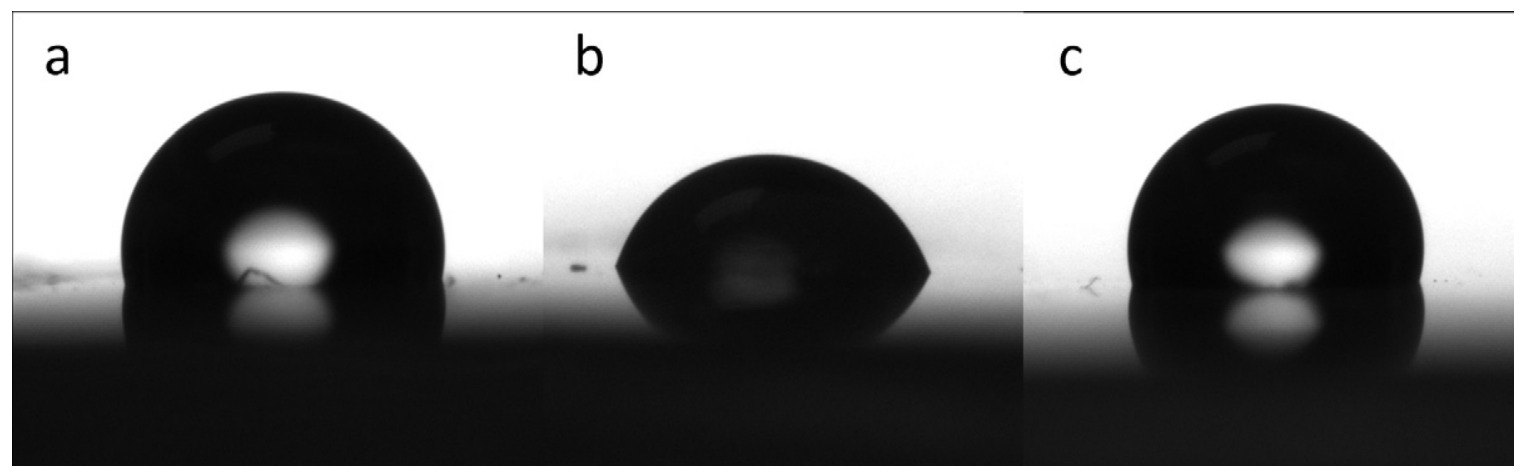

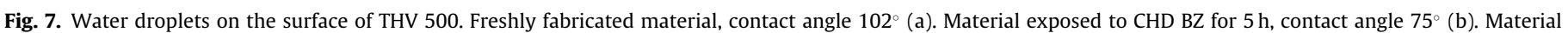

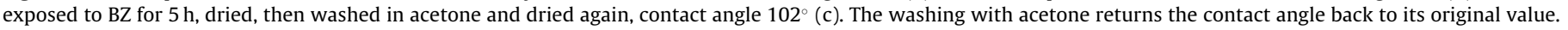
This observation suggests that acetone cleans the surface of the THV 500 structure. 


\section{Concluding Remarks}

Microfluidic devices hold promise for the production of heterogeneous, chemically complex molecular computing substrates. However, the high surface-to-volume ratio of microfluidic systems means that surface effects are a major issue-either in terms of the chemistry damaging the chip, or the chip surfaces affecting the chemistry. It becomes apparent that progress in this exciting area requires the co-development of device-substrate and reactionchemistry. While the developments in the area of microfluidics are largely driven by its commercial potential in healthcare applications, microfluidics offers also new possibilities for the design of molecular computing architectures. Such technical applications, however, differ in their requirements for the material of the microfluidic devices. We have surveyed recently introduced materials for their suitability in the technical setting of molecular computing and have identified a material compatible with the requirements of our BZ-droplet architecture. Our future work on droplet-compartmentalised BZ will concentrate on microfluidic devices from the fluoropolymer Dyneon THV 500, which we have shown here to be compatible with both the bulk oil phase and the BZ medium itself.

\section{Acknowledgement}

The research reported here is supported in part by Future and Emerging Technologies Grant FP7-248992 "NEUNEU" from the European Union.

\section{References}

Begolo, S., Colas, G., Viovy, J.-L., Malaquin, L., 2011. New family of fluorinated polymer chips for droplet and organic solvent microfluidics. Lab on a Chip 11 (3), 508-512.

Bonyár, A., Sántha, H., Ring, B., Varga, M., Gábor Kovács, J., Harsányi, G., 2010. 3D Rapid Prototyping Technology (RPT) as a powerful tool in microfluidic development. Procedia Engineering 5, 291-294.

Christopher, G., Anna, S., 2007. Microfluidic methods for generating continuous droplet streams. Journal of Physics D: Applied Physics 40 (19), R319.

Conrad, M., 1972. Information processing in molecular systems. Currents in Modern Biology (now BioSystems) 5, 1-14.
Corsi, J.C., King, P.H., Morgan, H., de Planque, M.R.R., Zauner, K.-P., 2011. BelousovZhabotinsky oscillations inside lipid-enclosed droplets. In: BioChemIT-1st COBRA Workshop on Biological and Chemical Information Technologies, Paris, France, Available from: www.cobra-project.eu/abstracts/corsi.pdf.

Duffy, D.C., McDonald, J.C., Schueller, O.J.A., Whitesides, G.M., 1998. Rapid prototyping of microfluidic systems in poly(dimethylsiloxane). Analytical Chemistry 70 (23), 4974-4984.

Gardner, J.W., Varadan, V.K., Awadelkarim, O.O., 2001. Microsensors, MEMS and Smart Devices. Wiley, Chichester.

Ginn, B.T., Steinbock, B., Kahveci, M., Steinbock, O., 2004. Microfluidic systems for the Belousov-Zhabotinsky reaction. The Journal of Physical Chemistry A 108 (8), 1325-1332.

Gorecki, J., Szymanski, J., Gorecka, J.N., 2011. Realistic parameters for simple models of the Belousov-Zhabotinsky reaction. The Journal of Physical Chemistry A 115 (32), 8855-8859.

Han, A., Graff, M., Wang, O., Frazier, A.B., 2005. An approach to multilayer microfluidic systems with integrated electrical, optical, and mechanical functionality. Sensors Journal, IEEE 5 (1), 82-89.

Haubert, K., Drier, T., Beebe, D., 2006. PDMS bonding by means of a portable, low-cost corona system. Lab on a Chip 6 (12), 1548-1549.

Huang, M.C., Ye, H., Kuan, Y.K., Li, M.H., Ying, J.Y., 2009. Integrated two-step gene synthesis in a microfluidic device. Lab on a Chip 9 (2), 276-285.

Huebner, A., Sharma, S., Srisa-Art, M., Hollfelder, F., Edel, J.B., deMello, A.J., 2008. Microdroplets: a sea of applications? Lab on a Chip 8 (8), 1244-1254.

Jones, G., Lovell, C., Morgan, H., Zauner, K.-P., 2011. Organising chemical reaction networks in space and time with microfluidics. International Journal of Nanotechnology and Molecular Computation (IJNMC) 3 (1), 35-56.

King, P., Covington, J., 2009. A novel monolithic microactuator fabricated by 3D rapid direct manufacture. Procedia Chemistry 1 (1), 1163-1166.

Kurin-Csörgei, K., Zhabotinsky, A.M., Orbán, M., Epstein, I.R., 1996. Bromate-1,4cyclohexanedione-ferroin gas-free oscillating reaction. 1. Basic features and crossing wave patterns in a reaction-diffusion system without gel. The Journal of Physical Chemistry 100 (13), 5393-5397.

Lee, J.N., Park, C., Whitesides, G.M., 2003. Solvent compatibility of poly(dimethylsiloxane)-based microfluidic devices. Analytical Chemistry 75 (23), 6544-6554.

Morel, M., Bartolo, D., Galas, J.-C., Dahan, M., Studer, V., 2009. Microfluidic stickers for cell- and tissue-based assays in microchannels. Lab on a Chip 9 (7), 1011-1013.

Theberge, A.B., Courtois, F., Schaerli, Y., Fischlechner, M., Abell, C., Hollfelder, F., Huck W.T.S., 2010. Microdroplets in microfluidics: an evolving platform for discoveries in chemistry and biology. Angewandte Chemie International Edition 49 (34), 5846-5868.

Wägli, P., Homsy, A., de Rooij, N.F., 2010. Norland optical adhesive (NOA81) microchannels with adjustable surface properties and high chemical resistance against IR-transparent organic solvents. Procedia Engineering 5, 460-463.

Xia, Y., Whitesides, G.M., 1998. Soft lithography. Annual Review of Materials Science $28,153-184$.

Zauner, K.-P., 2005. Molecular information technology. Critical Reviews in Solid State and Material Sciences 30 (1), 33-69. 